Etnográfica

Revista do Centro em Rede de Investigação em

Antropologia

vol. $14(2) \mid 2010$

Vol. $14(2)$

\title{
Transgression et désordre dans le genre : les explorateurs français aux prises avec les "berdaches" amérindiens
}

Gender disorder and transgression: french explorers wrestling with american "berdaches"

\section{Laurence Hérault}

\section{CpenEdition}

\section{Journals}

\section{Edição electrónica}

URL: https://journals.openedition.org/etnografica/316

DOI: 10.4000/etnografica.316

ISSN: 2182-2891

\section{Editora}

Centro em Rede de Investigação em Antropologia

\section{Edição impressa}

Data de publição: 1 junho 2010

Paginação: 337-360

ISSN: 0873-6561

\section{Refêrencia eletrónica}

Laurence Hérault, «Transgression et désordre dans le genre : les explorateurs français aux prises avec les " berdaches " amérindiens», Etnográfica [Online], vol. 14 (2) | 2010, posto online no dia 21 outubro 2011, consultado o 10 fevereiro 2022. URL: http://journals.openedition.org/etnografica/316 ; DOI: https://doi.org/10.4000/etnografica.316

\section{(c) (1) \&}

Etnográfica is licensed under a Creative Commons Attribution-NonCommercial 4.0 International License. 


\title{
Transgression et désordre dans le genre : les explorateurs français aux prises avec les " berdaches" amérindiens
}

\section{Laurence Hérault}

\begin{abstract}
A partir des textes des explorateurs français décrivant les " berdaches ", l'article essaie de comprendre la manière dont les cultures européennes ont conçu les expériences transgenres amérindiennes. Il montre notamment que l'étiquetage européen de la " transgenrité " amérindienne (sodomite/hermaphrodite) tient moins à une confusion face à une figure inconnue (un troisième sexe non répertoriée dans les cultures européennes), qu'à la reconnaissance du caractère transgressif d'une expérience qui renverse la hiérarchie des genres. Les " berdaches " posaient problème moins parce qu'ils contrevenaient à la bi-partition des genres ou encore à "l'hétérosexualité naturelle " comme peuvent nous le faire penser les textes au premier abord, mais bien plus parce qu'ils opéraient un retournement de prestige et de pouvoir culturellement inacceptable.
\end{abstract}

MOTS-CLÉS: berdache, transgenre, two-spirit, travestissement, homosexualité.

LA “ DÉCOUVERTE ” DE L'AMÉRIQUE A ÉTÉ POUR LES EUROPÉENS, ON le sait, une rencontre essentielle avec une " altérité " conçue comme radicale : le contact avec ces peuples aux "étranges " mœurs, via la constitution de la figure du "sauvage ", a bouleversé et a permis d'interroger nombre de conceptions occidentales. Parmi toutes les "bizarreries" aperçues alors dans les nations autochtones de l'Amérique du Nord, la rencontre de ceux qu'on a appelé " berdaches " semble avoir marqué les esprits et jeté un certain trouble parmi les observateurs. Ce trouble, éprouvé très tôt par les premiers explorateurs et colonisateurs, a concerné presque tous ceux qui les ont suivis quels que soient leur nationalité et leur statut. Au fil des siècles, nombreux sont ceux qui, au gré de leur contact avec les populations amérindiennes, ont rapporté leur désarroi dans leurs écrits, tel Edwin Denig, ce commerçant en fourrure qui 
note au milieu du $19^{\text {ème }}$ siècle son étonnement devant cette pratique qu'il rencontre chez les Crow : "strange country this, where the males assume the dress and perform the duties of females, while women turn men and mate with their own sex!" (Denig 1961: 187). Ces réactions européennes aux transgenres amérindiens sont d'ailleurs loin d'être toutes aussi civilement irritées que celle de Denig. Lire les récits des voyageurs, c'est être confronté, comme le signale Will Roscoe, non seulement à la stupéfaction, mais plus souvent encore à la consternation, à la répugnance et au dégoût le plus profond des auteurs. A l'égale de l'anthropophagie, à laquelle elle est d'ailleurs souvent associée dans les textes des conquistadores espagnols, la " berdachité " se donne comme une limite où se joue l'acceptabilité de l'Autre et apparaît aussi conjointement comme un outil très performant de justification du projet colonial et de la violence qu'il suppose. ${ }^{1}$

Devant ces réactions désapprobatrices et parfois violentes, on n'est guère étonné de constater que les anthropologues contemporains qui s'intéressent aux transgenres amérindiens jugent sévèrement les écrits des premiers voyageurs soulignant, comme Sabine Lang, par exemple, que ces textes, même lorsqu'ils ne mettent pas au premier plan les relations homoérotiques honnies, ont tendance à éclipser les descriptions détaillées des autres aspects du changement de genre. Ils sont donc non seulement toujours emprunts de jugement de valeurs mais en outre, et par le fait même, de peu d'utilité quant à notre connaissance du phénomène au moment de la rencontre avec les Européens (Lang 1998: 17-18). Will Roscoe, qui considère également que la littérature de contact sur les " berdaches "2 apporte plus de confusion que de compréhension, l'a cependant utilisée pour tenter de saisir le " fossé ” séparant les conceptions européennes et amérindiennes des genres et des sexualités (Roscoe 1998). Parcourant les textes à la recherche des termes utilisés pour catégoriser et définir les transgenres amérindiens, ${ }^{3}$ il montre ainsi que, contrairement

l La pratique de la sodomie est l'un des arguments avancés par Juan Ginés de Sepúlveda dans son traité des " justes causes de la guerre contre les indiens" (cf. Olivier $1990: 22$ ).

2 Le terme berdache s'est imposé pendant longtemps comme le terme générique permettant de désigner l'ensemble des transgenres d'Amérique du Nord. Dans les dernières décennies du siècle dernier, il a été vivement critiqué par les militants transgenres amérindiens en raison de son étymologie (voir note 17) et a été perçu comme éminemment disqualifiant et inapproprié. Le terme two-spirit lui a alors été préféré et est désormais largement employé dans les travaux sur la question. Dans ce texte, j'emploierai cependant le terme " berdache " dans la mesure où j'essaie justement de saisir la manière dont les Européens en sont venus à comprendre les transgenres d'Amérique comme tels. Mais je l'utiliserai entre guillemets de façon à signaler qu'il doit être uniquement entendu au regard du contexte historique de son apparition. Quant au terme transgenre, il est désormais assez souvent utilisé comme un terme transculturel utile pour qualifier des personnes qui se définissent de manière non-ordinaire au regard des classes de genre habituelles. C'est dans cette acception que je l'utiliserai ici.

3 Will Roscoe est, à ma connaissance, le seul à noter que les auteurs français, à l'exception de Deliette, n’ont pas utilisé le terme bardache dans leurs textes. 
aux langues autochtones qui avaient des termes particuliers pour qualifier les " berdaches ", les Européens ont éprouvé des difficultés pour désigner un rôle inconnu dans leurs sociétés et pour décrire l'ensemble des traits qui le composaient et qui en appelaient autant à l'économie et à la religion qu'au genre et à la sexualité. Incapables, donc, de saisir et de nommer ce " troisième genre ", 4 il montre ainsi comment les premiers voyageurs ont étiqueté certaines parties du rôle qui avaient du sens comparativement à l'expérience européenne, proposant une terminologie où dominent " hermaphrodite " et "sodomite ", chacun de ces termes fixant la "berdachité " dans l'ordre de l'anormalité sexuée ou sexuelle. Autrement dit, les Européens, incapables de saisir une expérience inédite, inconnue dans leurs propres sociétés, l'ont référée à des figures familières monstrueuses et/ou abhorrées, sur la base de certains aspects du comportement des " berdaches ". A travers cette incapacité à appréhender ce qui est présenté comme un système sexué alternatif (les cultures autochtones américaines accepteraient trois genres), les cultures européennes nous donneraient ainsi à voir leur attachement à un système plus restrictif des genres et des sexualités.

Sans aller totalement à l'encontre des idées qui viennent d'être exprimées, je voudrais cependant revenir sur cette incompréhension européenne. Je suis d'accord avec Lang, Roscoe, et bien d'autres encore, sur le fait que ces récits de voyage nous apportent peu sur la " transgenrité " amérindienne, et beaucoup sur la manière dont les cultures européennes ont conçu les expériences transgenres. Cependant, je ne suis pas sûre que les Européens ont catégorisé de manière réductrice les figures transgenres amérindiennes parce qu'ils ne comprenaient pas ce qu'ils voyaient. Je voudrais, au contraire, montrer que l'étiquetage européen de la "transgenrité " amérindienne tient moins à une confusion face à une figure inconnue, non répertoriée dans les cultures européennes, qu'à la reconnaissance du caractère transgressif d'une expérience particulière. En reprenant l'exploration d'un certain nombre de textes décrivant les “ berdaches ", 5 je voudrais essayer de montrer que la double catégorisation opérée n'est pas le fait d'une hésitation conceptuelle mais qu'on peut la lire comme une traduction pertinente du problème posé par les transgenres amérindiens

4 Roscoe fait partie des auteurs qui utilisent cette notion pour qualifier les transgenres amérindiens. Pour une critique de l'usage de cette notion en anthropologie, cf. Towle et Morgan (2006) ; Hérault (2007).

5 Pour plus de cohérence, j'ai retenu, essentiellement ici, les textes des voyageurs français. La plupart concerne plus particulièrement les Illinois, une population algonquine qui occupait, au moment de l'arrivée des Français en 1673, l'état actuel de l'Illinois et une partie des états voisins du Wisconsin, de l'Iowa et du Missouri (Marquette et Joliet 1681 ; Hennepin 1683, 1697, 1698 ; De Tonti 1697 ; St. Cosme 1861 [1700] ; Deliette 1934 [1704] ; Lahontan 1704 ; Charlevoix 1744 ; Bossu 1768). Certains textes évoquent d'autres populations et/ou réfèrent de manière plus générale aux " berdaches " présents dans les colonies françaises de l'époque (Laudonnière 1889 [1586] ; Le Moyne de Morgues 1990 ; Coréal 1722 ; Lafitau 1724). 
aux cultures européennes. Pour cela, je voudrais reprendre les textes des explorateurs à l'aune de ce que les historiens nous ont appris sur la question du genre à l'époque de la découverte et de la colonisation de l'Amérique. Si l'on veut, en effet, essayer de saisir ce que les explorateurs ont compris de ce qu'ils ont vu, il est important d'examiner avec attention ce qui leur a véritablement posé problème dans leur rencontre avec les " berdaches".

\section{LA DÉCOUVERTE DES IKONETA ILLINOIS : UNE RENCONTRE BIEN DÉRANGEANTE}

Jacques Marquette, jésuite français envoyé en 1673 explorer le Mississipi en compagnie de Louis Joliet, est le premier à relater l'existence de transgenres chez les Illinois :

Je ne sçay par quelle superstition quelques Illinois, aussi bien que quelques Nadoüessis, estant encore jeunes prennent l'habit de femme qu'ils gardent toute leur vie : il y a du mystère, car ils ne se marient jamais, \& font gloire de s'abaisser à faire tout ce que font les femmes; ils vont pourtant en guerre, mais ils ne peuvent se servir que de la massuë \& non pas de l'arc \& de la flèche, qui sont les armes propres pour les hommes; ils assistent à toutes les Jongleries \& à toutes les Dances solennelles qui se font en l'honneur du Calumet, ils y chantent mais ils n'y peuvent pas dancer ; ils sont appelez au Conseil, où l'on ne peut rien décider sans leur avis : enfin la profession qu'ils font d'une vie extraordinaire les fait passez pour des Manitous, c'est-à-dire de grands genies, ou personnes de consequence. (Marquette et Joliet 1681: 22-23)

Cette description des transgenres illinois est non seulement la première mais aussi la plus détaillée de toutes celles qui les concernent : y sont mentionnés le travestissement dès l'enfance, le célibat, l'adoption du rôle et des activités féminines, la participation “ à la manière des femmes " à la guerre (activité masculine valorisée), une fonction cérémonielle voire religieuse importante, un rôle politique et un statut valorisés. Dès cette première rencontre donc, le statut de ceux que les Illinois nomment ikoneta est bien documenté. ${ }^{6}$ On remarque également que Marquette n'assimile pas ce statut à ceux des hermaphrodites et des sodomites européens. Cette expérience semble ainsi à ses yeux à la fois

6 Le terme ikoneta est mentionné par Hauser (1990), mais ne figure pas dans Roscoe (1987). Aucun des textes mentionnés ici n'indique de termes vernaculaires, à l'exception du texte de Lahontan (1704) qui signale des "femmes de chasse " (ickoué ne kioussa), femmes dont la “ qualité ” de transgenres n'est cependant pas très claire. Dans la suite du texte, j'utiliserai le terme ikoneta pour référer à la conception autochtone qu'il est important de distinguer de la qualification européenne “ berdache " dont j'essaie ici de saisir les implications. 
inédite et étrange et il tente de la cerner et de la relater au plus près. Parce que les descriptions qui suivront se montreront moins attentives que la sienne, il est important de saisir l'étonnement de Marquette. Derrière son témoignage attentif, une interrogation et un malaise affleurent : ce statut l'étonne mais le dérange aussi. Il est non seulement " mystérieux " mais surtout incompréhensible : comment un homme peut-il trouver attrayant le statut et le rôle des femmes, comment peut-il "s'abaisser " à faire tout ce qu'elles font? Cette incompréhension semble d'ailleurs infléchir la description elle-même : Marquette a tendance à exprimer la spécificité du rôle en terme d'exclusion ou d'interdiction. Les ikoneta qui vont en guerre " ne peuvent" utiliser les armes des hommes, de même ils chantent mais " ne peuvent " danser lors de différentes festivités où leur participation semble cependant essentielle. Peut-être y a-t-il effectivement là une restriction autochtone, mais peut-être aussi un profond trouble de l'auteur face au travestissement et à l'inversion des rôles de genre observés.

Les pratiques de travestissement et même les histoires de changement de sexe ne sont pourtant pas inconnues en Europe. Le travestissement y est certes condamnable et condamné mais il n'est pas pour autant absent de l'expérience ordinaire. Il existe cependant une différence notable comparativement à l'attitude amérindienne : comme nous le montrent les travaux de Sylvie Steinberg, si le travestissement féminin bénéficie d'une indulgence relative, notamment lorsqu'il n'est pas associé à la " débauche " ou à l'homoérotisme, son homologue masculin est l'objet d'une forte réprobation. Par ailleurs, circulent sur le vieux continent un certain nombre de récits où des filles sont devenues garçons après avoir sauté une rivière ou s'être "échauffées " au cours d'une rencontre galante avec une autre jeune fille (Laqueur 1992 ; Steinberg 2001). Mais, là encore, il n'y a pas de transformation équivalente répertoriée pour les hommes.

Ces possibilités et la manière différente dont elles s'expriment pour les hommes et pour les femmes tiennent à la façon dont la différenciation des sexes est alors conçue. La distinction sexe/genre, telle que nous la concevons ordinairement, n'est pas ici opérante. Comme le souligne Thomas Laqueur, à cette époque, le sexe est avant tout une catégorie sociologique : "Etre homme ou femme, c'était tenir un rang social, une place dans la société, assumer un rôle culturel, non pas être organiquement l'un ou l'autre de deux sexes incommensurables" (1992:21-22). Autrement dit, le sexe n'est pas cette catégorie ontologique stable qui fonde l'identité sexuée d'une personne et que des comportements et des rôles sociaux viendraient traduire. Les médecins de la Renaissance, reprenant les analogies anatomiques de Galien, conçoivent, en effet, les sexes non pas comme deux catégories incommensurables mais bien plutôt comme deux versions différentes du même sexe. Ce modèle, que Laqueur a nommé le modèle unisexe, trace un continuum entre les corps 
mais ne postule pas pour autant leur égalité. ${ }^{7}$ Il est, bien au contraire, hiérarchisé puisque le corps féminin est envisagé comme une version moins parfaite du corps masculin : il a les mêmes organes que ce dernier mais au mauvais endroit. En outre, ces organes ont été comme stoppés dans leur croissance, par manque de chaleur : ce qui est achevé dans le corps viril, l'extériorisation des organes, reste en suspens dans le corps féminin. Par le jeu des températures et des tempéraments, la métamorphose du sexe est donc envisageable, mais elle l'est seulement dans le sens femme vers homme, c'est-à-dire celui de la perfection, comme le dit Gaspard Bauhin, un professeur d'anatomie de Bâle (1560-1624) : “Aussi ne trouvons-nous jamais en aucune histoire vraie qu'un homme soit jamais devenu femme, car Nature tend toujours vers ce qui est le plus parfait et non, au contraire, à opérer de telle manière que ce qui est parfait devienne imparfait" (in Laqueur 1992 : 146). Cette affirmation d'une transsexuation orientée reste cependant fragile du fait même de la possibilité de métamorphose. Le brouillage des genres, par exemple, fait craindre à certains médecins ou moralistes un changement de sexe susceptible d'invalider cette hiérarchie. On craint, par exemple, que les courtisans qui fréquentent assidûment les femmes ne deviennent comme elles et que cet efféminement ne les conduise à la déficience. Les conduites inappropriées, celles qui transgressent les codes du genre, peuvent donc se solder par un changement de sexe : en certaine circonstances le corps ne semble pouvoir résister aux pressions du genre brouillé et peut à tout moment se transformer pour s'accorder avec sa transgression (Laqueur 1992 : 139-152).

On comprend mieux alors l'inquiétude de Marquette : les Illinois qui paraissent endosser le genre féminin de manière sérieuse, radicale et durable mettent fondamentalement en question une hiérarchie des genres qui répond à l'ordonnancement du monde. Pour lui, ils désorganisent la distinction entre les genres et exposent virtuellement la perfection masculine au risque de la dissolution. Les ikoneta sont dévastateurs non pas parce qu'ils représenteraient une troisième classe de genre, antinomique avec le système duel européen mais parce qu'ils opèrent un retournement de prestige et de pouvoir culturellement inacceptable. ${ }^{8}$ Ce qui est déroutant pour les voyageurs

7 Ce continuum des corps, essentiellement anatomique selon Laqueur, est pour d'autres historiens plutôt humoral. Sylvie Steinberg, par exemple, souligne qu'à cette époque l'anatomie n'est probablement pas la manière la plus essentielle de définir la différence des sexes. Pour elle, l'anatomie se trouvait intégrée dans une conception humorale du corps plus large et plus englobante où chaque sexe avait ses propres caractères moraux mais aussi physiques (taille, couleur de la peau et des cheveux, forme des membres, aspect des chairs, timbre de la voix, etc.). Quoiqu'il en soit, dans les deux cas, il s'agit bien d'une biologie qui mobilise des explications différentes des nôtres surtout en ce qu'elle fait découler les caractéristiques physiques qui distinguent hommes et femmes d'une cosmologie plus générale.

8 Tout comme Pierre Aymond Dumoret, ce gentilhomme français (1678-1725) qui tentera en vain de faire accepter son désir de vivre en femme. Cf. l'analyse de sa dramatique histoire par Sylvie Steinberg (2001 : 91-126). 
européens, ce n'est donc peut-être pas tant l'existence d'un éventuel troisième genre, comme le supposent nombre d'auteurs contemporains; ce qui les déroute dans la manière d'être des ikoneta c'est qu'elle renverse les hiérarchies sociale et divine. En remettant en question la hiérarchie des genres, ces personnes mettent en péril l'ordre du monde. Il y a assurément de quoi être embarrassé et troublé.

\section{EXPLORATION DE L'EXPÉRIENCE TRANSGENRE :}

\section{DES SUPERSTITIONS AUX VICES}

Le texte de Marquette cherche moins à expliquer qu'à établir l'expérience transgenre dans tous ses aspects, mais il avance aussi un début d'explication : la transgenrité serait affaire de superstition. Ce lien se trouve aussi affirmé dans un autre texte, celui de Louis Hennepin, un missionnaire franciscain qui séjourne en Nouvelle-France entre 1675 et 1681 et qui écrit : "J'ay veu un garçon âgé d'environ dix-sept à dix-huit ans lequel avoit resvé qu'il estoit fille, il y ajoûta tellement foy qu'il croyoit estre tel ; il se vestoit comme les filles, \& faisoit tous les mesmes ouvrages que les femmes. " (1683:99) Pour les anthropologues contemporains cette mention d'un " rêve " qui amène à la transformation en femme évoque immanquablement l'un des modes de transsexuation largement répertorié parmi les populations amérindiennes : la confirmation du statut de transgenre par un être surnaturel lors d'une "vision " qui a lieu à l'adolescence, voire à l'âge adulte. ${ }^{9}$ L'individu ne peut se soustraire à cette vision qui l'amène parfois, en outre, au statut de "medicine person " (les manitous évoqués par Marquette). ${ }^{10}$

Cette piste "religieuse ", même dans son expression disqualifiante, ${ }^{11}$ pourrait offrir potentiellement un sens à cette transgenrité bizarre, lui donner quelques chances d'être intelligible. C'est ce que tente Lafitau qui, reprenant sans doute la description de Marquette, relie comme à son habitude ce travestissement amérindien aux pratiques religieuses antiques :

9 Callender et Kochems, qui tentent de caractériser les principaux traits de la transgenrité à partir d'une compilation des sources historiques et ethnographiques relatives à l'Amérique du Nord, retiennent la quête de vision comme l'un des modes privilégiés d'accès au statut de "berdache ". A propos des textes qui nous intéressent, ils pensent que la description de Marquette implique une telle vision même si elle ne la mentionne pas explicitement. De même, pour eux, le récit de Deliette qui n'évoque pas d'aspects surnaturels altère gravement la compréhension du rôle social du "berdache " (Callender et Kochems 1983: 451).

10 Dans certaines populations les rôles de transgenre et de " medicine person" sont étroitement associés, dans d'autres, ils ne le sont qu'éventuellement. Cf. Callender et Kochems (1983) ; Lang (1998) ; Williams (1986) ; ou encore Saladin d'Anglure (1986), qui décrit cette association chez les Inuits et l'interprète comme un révélateur de leur rôle doublement médiateur (ciel/terre, masculin/féminin).

11 Le chapitre dans lequel figure la description du jeune "berdache " dans l'ouvrage de Hennepin de 1698 a pour titre "superstitions des sauvages et leur créances ridicules". 
Chez les Illinois, chez les Sioux, à la Louisiane, à la Floride \& dans le Jucatan, il y a de jeunes gens qui prennent l'habit de femme qu'ils gardent toute leur vie, \& qui se croient honorez de s'abaisser à toutes leur occupations ; ils ne se marient jamais, ils assistent à tous les exercices où la religion semble avoir part, \& cette possession de vie extraordinaire les fait passer pour des gens d'un ordre supérieur, \& au dessus du commun des hommes. Ne serait-ce point les mêmes peuples que les asiatiques adorateurs de Cibèle, ou ces orientaux dont parle Julius Firmicus, lesquels consacraient, les uns à la déesse de Phrygie, les autres à Vénus Uranie, des prêtres qui s'habillaient en femmes, qui affectaient d'avoir un visage effeminé, qui se fardaient, \& déguisaient leur véritable sexe sous les habits empruntez de celui qu'ils s'efforçaient de contrefaire. (Lafitau 1724, t. 1 : 48-49)

Ce rapprochement, intéressant, reste cependant timide : contrairement à son habitude, Lafitau laisse ici la question de l'identité des coutumes en suspens. Plus tardivement dans son texte, lorsqu'il examine les cultes du feu, il affirme que ces " hommes déguisez en femmes \& qui font profession du célibat" ne sont pas commis à l'entretien du feu sacré (1724, t. 2 : 158). Autrement dit, les fonctions religieuses de ces " hommes travestis" semblent mal assurées ou difficilement soutenables. Lafitau nous livre lui-même les raisons de cette difficulté :

Quoique l'esprit de religion qui leur fait embrasser cet état les fasse regarder comme des hommes extraordinaires, ils sont néanmoins réellement tombez, parmi les sauvages même, dans ce mépris où étaient anciennement les prêtres de Vénus Uranie \& de Cybèle ; \& soit qu'effectivement ils se soient attirez ce mépris en s'asservissant à des passions honteuses, soit que l'ignorance des Européens sur les causes de leur condition, fondât contre eux des soupçons fâcheux ; ces soupçons entrèrent si avant dans leur esprit, qu'ils en imaginèrent tout ce qu'on pouvait penser de plus desavantageux ; \& cette imagination alluma si fort le zèle de Vasco Nugnes de Valboa capitaine espagnol qui découvrit le premier la mer du Sud, qu'il en fit périr un grand nombre, en lâchant sur eux ces dogues, furieux, dont ceux de sa nation se sont servis pour détruire une grande partie des Indiens. (1724, t. $1: 49-50)$

On perçoit ici la nature du problème de Lafitau dans la mesure où, derrière ce " célibat religieux" des transgenres amérindiens, pointe la question de leur sexualité : ces hommes travestis sont-ils sodomites ? La réponse de Lafitau est complexe et ambiguë comme on pouvait l'attendre d'un observateur attentif et passionné des mœurs amérindiennes et de la diversité culturelle. Sous sa plume, les pratiques sodomites sont tout à la fois réelles et fantasmées et leur 
condamnation, certes non négociable, offre des expressions contradictoires et gênantes : l'appréciation autochtone reste ambivalente associant considération et mépris ou bien se donnant comme une réaction à la condamnation européenne, elle-même parfois excessivement et injustement violente. Au bout du compte, il évite une prise de position qui pourrait le mettre en porte-à-faux tout en soulignant, de manière pertinente, l'importance de la question de la sexualité dans la compréhension européenne de la transgenrité amérindienne. Une lecture sexuelle de la transgenrité illinoise va en effet progressivement s'imposer et supplanter la piste religieuse comme on peut le voir dans le texte d'un autre jésuite, Pierre François-Xavier de Charlevoix : ${ }^{12}$

On a prétendu que cet usage venoit de je ne sçai quel principe de religion, mais cette religion avoit comme bien d'autres, pris sa naissance dans la dépravation du cœur, ou si l'usage, dont nous parlons, avoit commencé par l'esprit, il a fini par la chair : ces efféminés ne se marient point, \& s'abandonnent aux plus infâmes passions ; aussi sont ils souverainement méprisés. (Lettre de Juillet 1721, Charlevoix 1744: 4-5)

Ici la transgenrité n'est plus une absurde superstition " sauvage " mais se donne comme la dernière des perversions : l'invention de la figure du " berdache " est en cours.

\section{LA CONSTITUTION DU “BERDACHE ” : DES ILLINOIS ET DE LEUR "MALHEUREUX PENCHANT POUR LA SODOMIE"}

Si la sexualité des transgenres illinois, on l'a vu, est quasiment absente de la description de Marquette qui évoque simplement le célibat des ikoneta, elle va prendre une place grandissante dans les descriptions ultérieures. ${ }^{13}$ Louis Hennepin, dans un texte daté de 1697, offre une nouvelle description et surtout une autre explication de la transgenrité illinoise : ${ }^{14}$

Ils sont impudiques jusqu'à tomber dans le péché qui est contre nature. Ils ont des garçons, à qui ils donnent l'équipage de filles, par ce qu'ils les

12 Charlevoix fait deux séjours en Nouvelle France, le premier de 1705 à 1709 à Québec et le second de 1720 à 1722, durant lequel il voyage dans la région des grands lacs et descend le Mississipi.

13 Pour l'Amérique latine, il semble que cette lecture sexuelle de la transgenrité soit prépondérante dès le début de la conquête. C'est sans doute pourquoi R. Trexler (1995) privilégie une approche "homosexualisante" des "berdaches". Mais celle-ci semble moins pertinente pour l'Amérique du Nord dans la mesure où le comportement " homosexuel " apparaît comme une conséquence et non pas une cause du changement de genre. Cf. entre autres, Callender et Kochems (1983) ou Lang (1998). 14 On remarquera d'ailleurs que, contrairement à ses autres textes, celui-ci ne se donne pas comme le témoignage d'une rencontre personnelle. 
emploient à cet abominable usage. Ce garçons ne s'occupent qu'aux ouvrages des femmes, \& ne se meslent ni de la Chasse ni de la guerre. (Hennepin $1697: 219-220)$

Jean-François Buisson de St. Cosme, un jésuite de la " mission du Mississipi " mentionne également le " péché contre-nature ":

Nous vimes dans le village de Kappa un de ses malheureux qui s'habillant des leur jeunesse en fille servent au plus honteux de tous les vices mais cet infame n'étoit point de leur nation [Acanseas] il étoit Illinois parmy lesquels cela est tout commun. (St. Cosme 1861 [1700] : 42-43)

La description de Charlevoix, on l'a dit, inscrit elle aussi la transgenrité dans le registre des vices, puisque c'est sous cet intitulé qu'est livrée une description des ikoneta dans une lettre datée de 1721 :

Dans les pays méridionnaux ils gardent peu de mesures sur l'article des femmes, qui de leur côté sont fort lascives. C'est de là qu'est venuë la corruption des mœurs, qui depuis quelques années a infecté les nations septentrionnales. Les Iroquois en particulier étoient assez chastes avant qu'ils eussent commerce avec les Illinois, \& d'autres peuples voisins de la Louysiane : ils n’ont gagné à les fréquenter, que de leur être devenu semblables. Il est vrai que la molesse \& la lubricité étoient portées dans ces quartiers-là, aux plus grands excès. On y voyoit des hommes, qui n'avoient point de honte d'y prendre l'habillement des femmes, \& de s'assujettir à toutes les occupations propres du sexe, d'où s'ensuivoit une corruption qui ne se peut exprimer. (Lettre de Juillet 1721, Charlevoix 1744: 44-45)

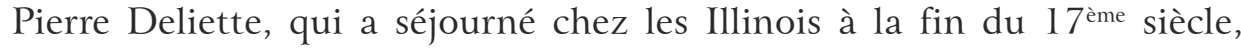
affirme également clairement un lien entre " péché de sodomie " et transgenrité mais avec une hésitation quant au sens du mouvement qui mène de l'un à l'autre :

Le péché de sodomie règne plus chez eux que dans aucune nations, quoy qu'il y ayent 4 femmes contre un homme, il est vray que quoy que débauchées elles gardent quelque mesure, qui empeschent les jeunes gens d'assouvir leurs passions autant qu'ils le souhaitteroient. Il y a des hommes qui sont élevez pour cela dès leur enfance, quand on leur voit prendre souvent la pioche, le fuseau, la hache et ne point se servir d'arc n'y de flèche, comme font tous les autres petits garçons, on leur met un morçeau de cuir ou d'éttoffe qui leur envelope depuis la ceinture jusqu'au genoux comme ont toutes les femmes, on leur laisse croistre les cheveux, qu'on attache par 
derrière et une petite peau qu'on leur met en bandoüillère qui est passée dessous un bras d'un costé et noüé sur l'épaule de l'autre, ils sont piqués aux joues comme les femmes sur le sein et sur les bras, et en contre font l'accent qui est différend de celuy des hommes, ils n'oublient rien pour ressembler aux femmes, il y a des hommes asses bruteaux pour en avoir avec eux, sur le méme pied, les femmes et filles qui se prostituent a ce malheureux sont des débordées. (Deliette 1934 [1704] : 329)

Pour Deliette donc, si les “ berdaches" montrent une inclination précoce pour le genre féminin en même temps qu'ils sont élevés pour servir de partenaires sexuels aux autres hommes, on découvre aussi finalement que leur sexualité ne se limite pas aux relations avec ces derniers : les femmes les fréquentent également. Chez De Lahontan, le lien avec les “ pratiques sodomites " n'est opéré que par la juxtaposition des phrases qui pourraient tracer aussi bien le portrait d'individus différents. Le travestissement et l'adoption des activités féminines ne sont pas non plus clairement mentionnés. ${ }^{15}$ Enfin Henri de Tonti remarque que les Illinois

[...] sont fort ardents pour les femmes, \& encore plus pour les garçons, aussi deviennent-ils tous presque effeminez par leur trop grande mollesse, \& par leur abandonnement au plaisir, soit que ce soit le vice du climat, soit que ce soit un effet de leur imagination pervertie. (De Tonti 1697 : 58-59)

Mais si la métamorphose des hommes en femmes est bien associée, pour lui, à cette sexualité “ débauchée ”, c'est d'une toute autre façon :

Ce qu'il y a de merveilleux en ceci, c'est que malgré ce malheureux penchant qu'ils ont pour ce vice infame, ils se font de très severes loix pour le punir : dès qu'un garçon est prostitué, il est dégradé de sa qualité d'homme, on lui défend d'en porter l'habit \& le nom, d'en faire la moindre fonction ; la chasse même lui est defenduë, on le renferme dans le rang \& dans l'occupation des femmes; celles-ci le haïssent autant que les hommes le méprisent, si bien que ces malheureux se voïent en même temps le rebut et l'opprobre de l'un \& l'autre sexe. (De Tonti 1697 : 59)

Pour De Tonti donc, la transgenrité est très nettement une sanction réservée à ceux qui se livrent au "vice infâme ". La " berdachité " n’est plus un choix ou une inclination de l'individu pour le genre féminin, elle est avant tout une exclusion du genre masculin : on " défend " à ce genre de garçons 
de se comporter en homme et on les " assigne " aux comportements féminins comme on les assignerait à résidence. ${ }^{16}$

Ce qui se déroule dans ces textes est fondamental pour la compréhension des transgenres amérindiens. C'est ici, en quelque sorte, que les ikoneta deviennent des bardaches, terme péjoratif qui désigne en français de l'époque les “ jeune[s] garçon[s] dont on abuse honteusement" (Richelet 1680: 65). Ce terme qui a été, en effet, appliqué par les voyageurs français aux transgenres illinois est à l’origine du terme générique " berdache " utilisé pour définir spécifiquement l'ensemble des transgenres amérindiens. ${ }^{17} \mathrm{Si}$ les textes évoqués ici n'utilisent pas, à l'exception de celui de Deliette,$^{18}$ le sans doute trop péjoratif bardache, on comprend bien que les descriptions qu'ils nous proposent du devenir "transgenre " servent bien à la métamorphose de l'ikoneta en bardache, c'est-à-dire à la conjugaison entre transgenrité et sexualité “ hors norme ”. Essayons

16 Un autre texte, plus tardif, celui de Jean-Bernard Bossu, fait de la " berdachité " une sanction autochtone. Mais contrairement à De Tonti, il ne s'agit pas là de condamner un "vice " sexuel mais une désertion au combat. Le principe est cependant comparable au sens où les hommes qui dérogent, dans des actes où la virilité est hautement engagée, sont déchus de leur qualité et ravalés à la condition inférieure des femmes: "Parmi les Sauvages [Illinois], ceux qui lâchent le pied, ou désertent dans une action où il s'agit de l'honneur, et de la défense de la patrie, ne sont point punis ; mais ils sont regardés comme l'opprobre du genre humain. Les autres leur reprochent toujours qu'ils ne sont point des hommes ; mais des vieilles. Ils sont méprisés des femmes mêmes, et les filles les plus laides n'en veulent point pour maris, et s'il arrivait que quelqu'une en voulu épouser un, les parents s'y opposeraient, dans la crainte d'avoir dans leur famille des hommes sans cœur ; et inutiles à la patrie. Ces sortes de gens sont obligés de laisser croître leurs cheveux, et de porter un alkonan [en note : petite jupe dont se servent les femmes sauvages pour cacher leur nudité] comme les femmes" (Bossu 1768 : 159-160).

17 Ce terme n'est pas propre au français mais se retrouve également dans les autres langues romanes avec le même sens. Il provient, via l'arabe, du persan bardeh utilisé pour désigner les esclaves et les prisonniers de guerre. Cette étymologie est connue depuis longtemps puisqu'on la rencontre dans une note des "Jesuit relations" en appendice d'un texte de Marquette (Thwaites 1900). Elle est reprise ensuite par les différents auteurs qui s'intéressent à la question à partir des années 1950 (Angelino et Shedd 1955; Callender et Kochems 1983; Jacobs, Wesley et Lang 1997; Lang 1998; Roscoe 1987; Williams 1986). Centrés sur l'importation américaine du terme, la plupart cependant se montre peu sensible à son importation européenne. Or celle-ci est tout aussi symptomatique des conceptions européennes de la sexualité des autres que l'est l'application américaine. Bardeh est, en effet, neutre et désigne tous les prisonniers qu'ils soient hommes ou femmes. En outre, en persan et en arabe, le terme ne comporte pas a priori de connotation sexuelle même si l'on peut penser que les esclaves et prisonniers assuraient, éventuellement ou probablement, un service sexuel. Dans les langues européennes, en revanche, la connotation sexuelle est primordiale mais l'idée d'infériorité sociale du bardache demeure cependant présente (soit du fait de son âge, de sa situation économique ou encore de son statut de prostitué). Létroite association dans les représentations européennes du Moyen Orient entre Islam, sodomie et violence semble avoir permis ce glissement sémantique. Cf., entre autres, Bleys (1995).

18 Et encore bardache ne figure-t-il pas dans le cadre de sa description des ikoneta mais alors qu'il décrit un jeu de ballon des Illinois où l'un d'eux sera blessé : «j'en ay vû dans cet etat qui se croyoient morts, les joueurs passent dessus sans en faire estat. [...] J'ay vû un bardache qui estoit à l'ecart comme les femmes, pour renvoyer la boulle du costé de son partis, suposé qu'elle fust a luy, a qui on donna la de la boulle dans un œil, qui sortit de la teste" (Deliette 1934 [1704] : 343). 
donc de comprendre comment celle-ci s'opère : quelles sont les raisons qui peuvent rendre compte de la reconnaissance des ikoneta comme bardaches?

On pourrait penser, au premier abord, qu'ils sont reconnus tels en raison de leur pratique homoérotique, mais cette dimension, sans doute réelle, de leur pratique sexuelle est loin de rendre compte, à elle seule, de cette qualification. Tout d'abord les textes ne limitent pas " le péché infâme " aux seuls ikoneta. On voit bien, au contraire, que c'est, à chaque fois, l'ensemble de la population masculine qui est concernée par cette accusation et que celle-ci figure, en outre, dans une évocation plus générale de la transgression sexuelle. Les amérindiens et particulièrement les Illinois, ont, selon nos auteurs, une sexualité quelque peu débridée qui les conduit couramment à l'adultère et à la sodomie. Une telle accusation de sodomie figure dans le discours occidental sur l'autre bien avant la découverte de l'Amérique ${ }^{19}$ mais elle trouve là un support particulièrement efficient, notamment en Amérique latine où elle sert le projet colonial européen. ${ }^{20} \mathrm{Si}$ cette accusation de sodomie appartient à une réthorique coloniale bien répertoriée, reste que dans les textes cités, une différence est opérée par les auteurs entre " travestissement" et sodomie : selon eux, tous les sodomites ne se prêtent pas à la métamorphose du genre, seuls certains s’y soumettent ou s'y inscrivent. Les comportements des ikoneta doivent être spécifiés car ils débordent l'accusation traditionnelle de barbarisme sodomite.

Cette spécificité pourrait tenir à une sorte d'exclusivité dans le choix des partenaires sexuels : parmi les " libertins" illinois aux pratiques largement " bisexuelles", seuls ceux qui sont portés exclusivement vers des partenaires masculins seraient reconnus comme bardaches. Cependant, si l'on en croit Deliette, les ikoneta n'ont pas une pratique exclusivement homoérotique : leur désir les conduit aussi vers les femmes, ce qui ne semble d'ailleurs pas les racheter aux yeux de notre explorateur. Si une association est faite entre transgenre et " pratique contre-nature " ce n'est donc pas en raison d'un choix particulier de partenaire sexuel comme nous serions tentés de le croire étant donnés nos catégories usuelles d'homosexualité et d'hétérosexualité. Une autre explication de cette association étroite entre transgenrité et "sodomie " pourrait tenir, non plus à la pratique amérindienne, mais à l'expérience européenne : dans "l'Ancien Monde ", les " sodomites" seraient invariablement travestis ou efféminés. Mais là encore si les moeurs françaises de l'époque offrent de nombreux exemples d'association entre travestissement et " transgression sexuelle ", il n'y a pas, pour autant, d'assimilation systématique entre pratique homoérotique et travestissement. Il y a, certes, des moralistes pour relier le travestissement des hommes aux "pratiques infâmes ". Mais il y a aussi des témoignages, tel celui de l'abbé de Choisy, qui montre que le libertinage se divertit d'un 
travestissement complexe à orientation hétéroérotique: l'amant se présentant dans des vêtements féminins quand sa partenaire endosse ceux propres aux hommes. Il existe également une littérature importante dans laquelle le travestissement est utilisé dans le jeu de séduction mais où, grâce au retournement de situation, il ne franchit pas les frontières de la sexualité (Steinberg 2001). S'il est ainsi avéré que travestissement et " débauche " sexuelle cheminent parfois de conserve, l'expérience ordinaire ne voit pas là une liaison stable : on sait que l'inconduite sexuelle s'exprime bien en dehors de toute "mascarade " vestimentaire, de même que le travestissement s'affranchit assez souvent de la sexualité homoérotique et même de la sexualité en général. Comme le rappelle Steinberg, “ pour l'époque moderne, il n'y a aucun lien simple et ce, chez les hommes comme chez les femmes, entre le travestissement et la (ou les) transgression(s) sexuelles(s) " (2001 : 124). Autrement dit, la réalité américaine confronte les voyageurs français à une situation bien semblable à celle qu'ils connaissent : ici et là-bas, tous les " sodomites " ne sont pas travestis ni tous les travestis " sodomites".

Le travestissement n'est pas le seul mentionné, dans les textes cités, la dimension plus floue de l'efféminement est également présente mais dans une acception très différente de la nôtre que le texte de De Tonti permet d'apercevoir. Son récit rend, en effet, plus explicite les rapports complexes et divers qui lient alors effémination et sexualité. Pour De Tonti, les Illinois ne "s'efféminent " pas parce qu'ils se livrent à des relations homoérotiques comme un lecteur contemporain serait tenté de le penser. De Tonti distingue deux choses : d'une part l' " efféminement" et d'autre part la " berdachité ". Pour lui, c'est autant la fréquentation assidue des femmes que des garçons qui amènent les hommes à la "mollesse " féminine. En fait, les jeunes garçons sont proches des femmes en ce qu'ils sont encore marqués par une enfance qu'on rapproche, alors, de la féminité : les petits enfants, tout comme les femmes, sont des êtres imparfaits. Leur devenir cependant les distingue : en grandissant, les garçons se perfectionnent tandis que les filles restent toujours limitées en raison de leur déficience initiale (Steinberg 2001 : 105-109). Si les Illinois "s'amollissent " et "s'efféminent " c'est donc parce qu'ils se montrent attirés, de manière générale, par les " êtres féminins ". Cette effémination, certes dommageable, n'est cependant pas véritablement condamnable comme l'est, pour De Tonti, le comportement des ikoneta.

Il semble en fait que pour comprendre la reconnaissance des ikoneta comme bardaches, il faut examiner de plus près, les comportements sexuels qu'on tient alors pour transgressifs. À cette époque les pratiques “ contre-nature " ne désignent pas simplement, en effet, les comportements homoérotiques. La transgression sexuelle renvoie à trois types d'opposition : légitime/adultère, fécondité/infécondité, activité/passivité. Les relations sexuelles entre hommes contreviennent diversement à cet ordonnancement : elles sont fondamentalement infécondes, 
éventuellement adultères si l'un au moins des partenaires est marié, mais pas nécessairement transgressives du point de vue des rôles sexuels assumés. Les hommes qui ont des relations avec d'autres hommes sont, en effet, distingués selon la manière dont ils prennent part à la relation sexuelle : ceux qui sont présumés “ actifs" sont nommés bougres quand ceux qui endossent ce qu'on pense comme le rôle " passif " sont dits bardaches (Courouve 1985: 59-65, 70-78). Dans les définitions de ce dernier terme, cette " passivité " sexuelle est en outre associée à la jeunesse de l'individu et, plus ou moins explicitement, à son infériorité sociale et/ou économique. Cette catégorisation, on le voit, ne renvoie pas explicitement au travestissement ni à une mise en acte des rôles sociaux féminins mais elle s'inscrit en revanche dans une hiérarchisation des rôles sexuels qui recoupe la hiérarchisation des genres puisque la " passivité " et "l'activité " sexuelles sont respectivement conçues comme féminine et masculine. C'est sans doute en cela que l'ikoneta illinois devient semblable au bardache français pour ceux qui le rencontrent. Au-delà d'un engagement dans des relations homoérotiques qui ne les spécifie pas, ce qui les identifie, c'est le fait qu'ils transgressent les frontières des genres puisqu'ils assument véritablement un rôle réservé aux femmes que ce soit socialement et/ou sexuellement. En outre, dans ces textes qui privilégient une lecture sexuelle de la transgenrité amérindienne, les ikoneta sont décrits comme appartenant à une classe inférieure : ils ne sont plus des hommes qui choisissent par superstition ou extravagance un statut particulier valorisé mais des garçons méprisés et exploités à l'instar des bardaches. ${ }^{21}$ Cette transgression de la hiérarchie des genres est donc bien ce qui les rapproche définitivement, bien plus que l'homoérotisme dont on a vu qu'il était en Europe et en Amérique plus extensif, ou que le travestissement et l'efféminement qui ne leur sont pas non plus spécifiques.

Ainsi, les transgenres amérindiens sont reconnus comme bardaches moins parce que les voyageurs européens se focalisent sur leur pratique sodomite que parce qu'ils reconnaissent dans les manières d'être, finalement différentes, des ikoneta et des jeunes sodomites européens une même charge transgressive. Autrement dit, ce que les voyageurs voient c'est une version " renversante " de la hiérarchie des genres et non pas une forme "alternative" du système des genres. Le problème posé par les transgenres ne tient pas tant au fait que la conception européenne bi-partite des genres est confrontée, à travers eux, à une conception tripartite inédite et incompréhensible. Ce qui est problématique ici, c'est que cette conception des genres s'affronte à l'impensable : la violation de l'ordre des genres inscrit dans la cosmologie générale. En se comportant sexuellement et socialement comme des femmes, les ikoneta vivent en dessous de leur condition et menacent directement l'ordre social. On va

21 Will Roscoe (1998) a noté dans d'autres textes cette tendance à décrire l'expérience " berdache " par des tournures passives qui la font apparaitre comme quelque chose d'essentiellement subi. 
voir d'ailleurs que lorsque ce renversement n'est pas patent, le " problème " du "troisième genre " n'en est pas un pour les voyageurs français. Pour cela, il nous faut quitter, pour un temps, le pays illinois pour nous intéresser à la Floride.

\section{LA DÉCOUVERTE DES HERMAPHRODITES DE FLORIDE : UNE CURIOSITÉ DIGNE D’ÊTRE RAPPORTÉE}

Dans un certain nombre de textes d'explorateurs de l'époque, comme l'a souligné Will Roscoe, le terme "hermaphrodite " côtoie assez fréquemment celui de "sodomite". Mais cette juxtaposition est tardive dans la rencontre entre Français et " berdaches " : les transgenres ont d'abord été vus, par les premiers Français, uniquement comme des hermaphrodites. Contrairement à leurs successeurs, les Français qui débarquent en Floride ${ }^{22}$ dans les années 1560 ne voient pas d'hommes "efféminés " mariés à d'autres hommes. René de Laudonnière et Jacques Le Moyne de Morgues rencontrent, en revanche, quantité d'hermaphrodites durant leur séjour: " Il y a dans tout ce pays beaucoup d'hermaphrodites, qui se donnent la plus grande peine et portent les victuailles quand ils vont à la guerre ", écrit Laudonnière qui mentionne, en outre, plusieurs rencontres avec ces derniers que l'on peut apparemment confondre avec des femmes: "Nous rencontrâmes une femme indienne de grande stature qui était aussi un hermaphrodite, qui vînt à notre rencontre avec un grand récipient plein d'eau claire avec laquelle elle nous rafraîchit grandement " (Laudonnière 1889 [1586]: 414). Son compagnon de voyage qui réalisera, par la suite, un portrait de ces hermaphrodites note quant à lui:

Les hermaphrodites, un mélange des deux sexes, sont fréquents ici, et les indiens les utilisent à la place des bêtes pour porter les charges car ils sont robustes et forts, bien qu'ils soient odieux aux indiens eux-mêmes. Quand un indien meurt de blessure ou de maladie, deux hermaphrodites [...] [s'occupent des soins au mort et de son enterrement]. Les personnes affectées par des maladies contagieuses sont aussi portées sur le dos par des hermaphrodites en des lieux convenus où on leur donne le nécessaire et où l'on prend soin d'eux jusqu'à ce qu'ils aient retrouvé la santé. (Le Moyne de Morgues 1990: pl. XVII)

Dans ces récits, ce qui caractérise les hermaphrodites et les distingue des autres, c'est avant tout leur position sociale servile. Les auteurs insistent sur

22 Le terme Floride désigne à l'époque l'ensemble de la région sud-est de l'Amérique du Nord ; cependant Laudonnière et Le Moyne de Morgues débarquent dans l'actuelle Caroline du Sud. Cf., pour plus de précisions, Lhoumeau (2000). 
leur statut social prenant soin de décrire attentivement les tâches et les devoirs qui y sont associés. Comparativement au texte de Marquette qui n'utilisait aucun terme spécifique pour désigner les transgenres illinois, l'usage du terme hermaphrodite est ici référé à l'existence d'une troisième classe sexuée distincte de celles des hommes et des femmes. Eu égard au système de genre européen, on pourrait donc s'attendre à ce que nos explorateurs soient, ici aussi, décontenancés par la constitution de cette classe sexuée alternative voire indignés par l'infériorité statutaire des hermaphrodites. Pourtant leur relation n'est guère désapprobatrice, elle serait même presque admirative compte tenu du rôle utilitaire de ces êtres ambigus. Pour saisir cette relative bienveillance, il faut remarquer, en premier lieu, que nos explorateurs semblent en terrain familier quant à la compréhension du genre : il leur semble qu'en Amérique aussi les genres, même en surnombre, sont des catégories sociologiques qui classent et hiérarchisent les individus. Autrement dit, comme sur le vieux continent, le problème posé par les hermaphrodites n'est pas un problème de "sexe " mais de "genre ". En Europe, l'ambiguïté des hermaphrodites pose bien évidemment question : là, comme ailleurs, il convient de savoir comment il est possible de les considérer. Afin de statuer sur leur sort, la médecine légale s'intéresse à leur corps, mais cette investigation n'a pas pour objectif de découvrir leur "vrai sexe ", elle s'attache plutôt à savoir " vers quel genre l'architecture de leur corps les inclin[e] le plus volontiers " (Laqueur 1992 : 153). L'important est, en effet, de préserver les catégories de genre et donc de déterminer dans quelle mesure ces individus et leurs organes sont aptes à tenir les rôles de genre propres aux hommes ou aux femmes notamment dans l'ordre de la sexualité. Il est essentiel, en effet, que nul ne vive en-dessous ou au-dessus de sa condition, c'est-à-dire que chacun soit à la place qui lui revient légitimement. La solution européenne au problème hermaphrodite se différencie donc de la solution américaine en s'orientant vers un reclassement (l'hermaphrodite est considéré comme homme ou comme femme) plutôt que vers la création d'une classe spécifique (comme le rapporte les auteurs). Mais si la manière amérindienne de classer les androgynes est inédite pour nos explorateurs (et donc digne d'être rapportée), elle ne se distingue pas fondamentalement du reclassement européen : il s'agit bien, dans les deux cas, de définir le statut de genre des androgynes. En outre, la classification amérindienne, en infériorisant très radicalement les hermaphrodites, semble se montrer aussi sourcilleuse que l'européenne quant à la hiérarchie des genres. Autrement dit, la reconnaissance d'une classification tripartite des genres serait moins dérangeante pour nos explorateurs qu'on aurait pu le penser : dans la mesure où elle ne renverse pas l'ordre sexué, elle semble compréhensible voire même acceptable. Parce que les hermaphrodites de Floride, contrairement aux ikoneta illinois, sont politiquement et socialement insignifiants, leur existence ne met pas en danger les conceptions européennes. 


\section{HERMAPHRODITES ET SODOMITES : DE LIDENTITÉ DES “ BERDACHES ”}

Cet usage " premier " et isolé du terme hermaphrodite par Laudonnière et Le Moyne de Morgues se distingue de ceux qui seront faits plus tardivement. En effet, dans les textes postérieurs qui décrivent les Illinois, le terme hermaphrodite côtoie le plus souvent le terme sodomite. Cette nouvelle présence peut s'expliquer en partie par un souci de référence aux écrits des découvreurs de la Floride. La mention des hermaphrodites apparaît, en effet, essentiellement sous une forme stéréotypée (qui insiste sur l'abondance de ces êtres), et semble, en outre, assez souvent surajoutée à la description ${ }^{23}$ si bien qu'on peut voir là un jeu d'intertextualité : les auteurs essaient d'ajuster leur description concernant les Illinois à ce que d'autres ont vu en Amérique en d'autres temps et en d'autres lieux. Il n'est d'ailleurs peut-être pas étonnant de constater que les textes où les hermaphrodites sont présents sont également ceux qui sont considérés comme plus ou moins apocryphes par les historiens : De Tonti, qui a bien résidé en pays Illinois aux côtés de De La Salle, n’a pas reconnu, par la suite, le texte qui lui est attribué et qui aurait été rédigé à partir de l'un de ses mémoires. Hennepin est considéré, par ses contemporains même, comme un quasi affabulateur : on sait du moins qu'il s'est attribué des exploits qu'il n'a pas réalisés (Hauser pense que son texte de 1697, dans lequel figure la mention des hermaphrodites, est issu d'un manuscrit d'un autre franciscain, Zénobius Membré ; cf. Hauser 1990 : 60, note 5). Enfin, il n'est pas sûr que Lahontan ait visité le pays illinois, ce que pourrait suggérer sa description un peu hétéroclite.

Parfois, cependant, l'usage du terme hermaphrodite nous amène au-delà de cet aspect purement référentiel, et notamment vers une nouvelle dimension de la conception européenne de la transgenrité amérindienne. Il semble, en effet, qu'on puisse lire en germe, dans certains textes du $18^{\text {ème }}$ siècle, une conception

23 "Ils sont fort ardents pour les femmes, \& encore plus pour les garçons, aussi deviennent-ils tous presque effeminez par leur trop grande mollesse, \& par leur abandonnement au plaisir, soit que ce soit le vice du climat, soit que ce soit un effet de leur imagination pervertie. On remarque parmi eux grand nombre d'Hermaphrodites. Ce qu'il y a de merveilleux en ceci, c'est que malgré ce malheureux penchant qu'ils ont pour ce vice infame, ils se font de très severes loix pour le punir. " (De Tonti, 1697, 58-59, italiques ajoutés)

"Les Hermaphrodites sont en grand nombre parmi eux. Ils ont ordinairement plusieurs femmes, \& prennent souvent toutes les Sœurs, disans qu'elles s'accordent mieux que des étrangères. Cependant ils sont si jaloux, qu'ils leur coupent le nez sur le moindre soupçon. Ils sont impudiques jusqu'à tomber dans le péché qui est contre nature." (Hennepin 1697 : 219-220, italiques ajoutés)

"Il y a des Sauvages qui observent le Celibat jusqu'à la mort, \& qui ne vont jamais à la guerre, ni à la chasse, parce qu'ils sont ou lunatiques, ou incommodez; quoi qu'il en soit, on a pour eux autant de consideration que pour les plus sains \& les plus braves du Païs, \& si l'on en fait quelques railleries, ce n'est jamais en leur presence. L'on trouve parmi les Ilinois quantité d'Hermaphrodites; ils portent l'habit de femme, mais ils font indifferemment usage des deux Sexes. Ces Ilinois ont un malheureux penchant pour la Sodomie, aussi bien que les autres Sauvages qui habitent aux environs du Fleuve de Missisipi." (Lahontan 1704 : 141-142, italiques ajoutés) 
identitaire plus que statutaire de cette "infâmie ". Un passage des Voyages de François Coréal aux Indes occidentales est intéressant à cet égard : son auteur, "obligé ” de synthétiser les descriptions de ses prédécesseurs, s'interroge sur l'adéquation du terme hermaphrodite. ${ }^{24}$ Sa relation sur les transgenres mêle, comme d'autres, des informations en provenance de plusieurs des textes rencontrés ici :

Les hommes sont fort enclins à la sodomie ; mais les garçons qui s'abandonnent ainsi sont exclus de la société des hommes, \& envoiés à celles des femmes, comme étant effeminés. Ils y sont confondus parmi les Hermaphrodites, qu'on dit se trouver en quantité chez les Floridiens. Je crois que ces Hermaphrodites ne sont autres que des garçons effeminés, qui en un sens sont de veritables Hermaphrodites. Quoiqu'il en soit, on les emploie tous à divers ouvrages de femmes, à des fonctions serviles \& à porter les munitions de bouche \& les provisions de guerre. Ils sont aussi distingués des hommes \& des femmes par la couleur des plumes qu'ils se mettent sur la tête, \& par le mépris qu'on fait d'eux. (Coréal $1722: 29$ )

Ce texte est fascinant par sa tentative de rendre en un ensemble cohérent une foule d'informations de provenances diverses et surtout assez souvent contradictoires. Il doit ainsi affronter ce que les autres auteurs laissent en suspens : quel lien établir entre hermaphrodites et " garçons sodomites" ? Son interprétation est intéressante en ce que, successivement, elle les distingue en les associant (les garçons efféminés sont confondus parmi les hermaphrodites), les assimile (ils ne sont autres que) et finalement les identifie par un recours au qualificatif "véritable ". Contrairement à ce que nous avons vu chez Laudonnière et Le Moyne de Morgue, l'usage que fait Coréal de la catégorie hermaphrodite est ici moins un usage sociologique qu'ontologique. Ce terme ne lui sert pas à désigner/décrire des individus appartenant à une classe particulière, bien au contraire, cette notion l'entraîne dans une discussion sur l'identité des transgenres. Le terme hermaphrodite lui sert à répondre à une question identitaire : quelle sorte d'êtres sont ces gens ? La réponse de Coréal est complexe et difficile à saisir mais on peut peut-être y voir l'émergence d'une affirmation de la nature particulière des transgenres. Dire in fine, comme il le fait, que ces " garçons sodomites" sont véritablement des androgynes, n'est-ce pas dire qu'ils ont une " nature " différente de celles des hommes et des femmes et non pas seulement un statut différent ? Pour Coréal, les " berdaches" seraient des êtres singuliers et leurs comportements seraient à relier à cette configuration identitaire spécifique.

24 Ce texte est vraisemblablement apocryphe. Il est dit être traduit de l'espagnol alors qu'aucun original dans cette langue n'est connu et l'identité même de l'auteur est questionnée. C'est d'ailleurs en ce sens que sa tentative de synthèse est intéressante pour nous. 
Cette compréhension nouvelle des "berdaches " qui paraît émerger chez Coréal est sans doute plus évidente à saisir dans un texte de Cornelius de Pauw de 1770. Ce dernier, qui n'est pas un voyageur, s'interroge, en effet, sur l'existence des hermaphrodites de Floride à une époque où le " modèle unisexe " tend à être supplanté par un modèle à deux sexes incommensurables. A la fin du $18^{\text {ème }}$ siècle s'affirme, en effet, une conception ontologique de la différenciation des sexes qui se donne à voir, selon Laqueur, dans la manière dont les organes sont vus et conçus. Dans le modèle téléologique mâle, les organes sont semblables mais différemment disposés et inégalement parfaits, et fonctionnent essentiellement comme des signes du statut sexué des personnes. Dans le nouveau modèle, le sexe est ontologique avant d'être social et les organes deviennent des preuves de la nature spécifique des individus. En conséquence, la manière de concevoir et de traiter les hermaphrodites se modifie d'un modèle à l'autre. Dans le premier cas, les organes servent à décider de l'appartenance légitime de l'individu à l'une des deux catégories de genre; dans le second ils doivent dire quel est le sexe véritable de la personne. En outre, les organes masculins et féminins étant désormais incommensurables, l'androgynie est de plus en plus conçue comme une impossibilité. Au $18^{\text {ème }}$ siècle, la plupart des auteurs considèrent ainsi que les hermaphrodites n'existent pas ; ce sont simplement des hommes ou des femmes ayant une conformation physique anormale, qu'on a pris pour androgynes par méconnaissance et erreur de jugement. C'est dans ce contexte que De Pauw tente, lui aussi, de répondre à la question qui traverse le texte de Coréal : qui sont ces êtres-efféminés-sodomites-hermaphrodites?

De Pauw examine successivement les différentes interprétations de nos voyageurs. Il rejette d'abord "l'interprétation sodomite " des jésuites, car s'il ne doute pas de la "corruption du goût et de l'instinct " des Américains, cette débauche probable ne lui semble pouvoir s'associer ni logiquement avec la servilité, ${ }^{25}$ ni nécessairement avec le rôle de genre féminin :

[...] il serait contradictoire de maltraiter si injurieusement ceux qui auraient tant de droit à la reconnaissance : car enfin tous les hommes vicieux ne sont pas des hommes ingrats. On ne comprend pas d'ailleurs pourquoi des sauvages, adonnés à de telles débauches, seraient obligés de prendre des accoûtrements de femmes. (De Pauw 1770 : 102)

Il balaye également les affirmations de ceux qui ne voient là que des hommes travestis : il met en cause, d'une part, leur expertise médicale - " on pourrait demander à un voyageur qui parle si pertinemment, s'il a eu assez de crédit, ou d'autorité pour se faire montrer les parties sexuelles de ces êtres incertains,

25 Il suppose, comme la plupart des voyageurs l'y invitent, que les hermaphrodites sont socialement infériorisés et méprisés. 
\& si avec cela les connaissances anatomiques ne lui ont pas manqué pour juger du degré de leur hermaphroditisme ?" (1770 : 103) -, et d'autre part, il souligne l'impossibilité, pour des hommes normaux, à déroger volontairement à leur statut et à leurs prérogatives :

[l'auteur] aurait du dire pourquoi on voit entre les indigènes de la Louisiane, des hommes qui nés aussi libres que leurs compatriotes, consentent néanmoins à passer, toute leur vie, pour femmes \& qui s'acquitent volontairement des devoirs réservés au dernier des esclaves. (De Pauw 1770 : 103)

La réponse qu'il propose associe étroitement le " consentement à la féminité et à la servilité " aux particularités biologiques de ces êtres. En se basant sur un récit qui affirme que les femmes de Floride se font toutes circoncire, il suppose que l'excroissance du clitoris est chose commune en Amérique et que les transgenres "sont des femmes en qui ce défaut ne se corrigeait pas " (1770 : 105); femmes que pour cette raison on condamnait à une servitude perpétuelle les considérant “ comme des individus d'une nature inférieure \& d'une race abatardie " (1770 : 105). L'explication fournie par De Pauw montre bien le renversement qui s'opère au $18^{\text {ème }}$ siècle : dans cet " organe saillant " des transgenres, il ne voit pas un organe architecturalement proche d'un pénis (ce qui dans une logique ancienne amènerait une discussion sur la possibilité de classer son possesseur dans le genre masculin) ; il n'aperçoit qu'un clitoris anormal, c'est-à-dire un organe spécifiquement féminin mais monstrueux. La nature féminine des transgenres n'est donc pas discutable et leur infériorisation sociale semble logique au regard de leur difformité constitutionnelle. Autrement dit, pour De Pauw il n'y a qu'une seule explication rationnelle à leur infériorité et elle tient à leur nature biologique défaillante.

Cette vision ontologique des sexes n'est certes pas si flagrante dans les textes des voyageurs de la première moitié du $18^{\text {ème }}$ siècle, mais on peut se demander si elle n'y est tout de même pas balbutiante dans la mesure où leur usage du terme hermaphrodite invite, comme chez Coréal, à une interrogation sur l'identité des transgenres. Dans la plupart des textes qui mentionnent si anachroniquement l'existence d'hermaphrodites au milieu d'une description des "garçons sodomites", il n'est pas invraisemblable de penser que cette intrusion ait, entre autres fonctions, d'asseoir l'idée d'une personnalité originale : évoquer les hermaphrodites au moment où l'on est en train de constituer la figure du " berdache ", ce peut être confirmer leur spécificité. Si tout un chacun peut devenir efféminé (trop grande proximité avec les êtres féminins) ou sodomite (abandon coupable au péché), tout le monde ne peut devenir " berdache ", ce sont là des êtres particuliers. La " berdachité " ne ressort pas de cette interprétation comme une configuration moins répréhensible, mais on sait du moins qu'elle n'est ni commune ni ordinaire. L'utilisation 
conjointe de ces deux catégories est ainsi peut-être moins la marque de l'hésitation des auteurs devant une figure qui n'est pas répertoriée dans l'expérience européenne qu'une manière d'affirmer qu'il y a là une transgression qui tient à des propriétés personnelles plus qu'à une dérive comportementale potentiellement attrayante. Le transgenre amérindien, parce qu'il défie l'orthodoxie du genre, non seulement dans le cadre sexuel mais dans tous les contextes sociaux, en vient à mettre en porte-à-faux l'idée selon laquelle la transgression sexuée et sexuelle est virtuellement l'affaire de tous. En se donnant non seulement comme homologue authentifié du " sodomite passif", mais surtout comme une figure spécifique voire typique, il tend à affirmer, ou du moins à suggérer, que cette transgression-là est caractéristique d'une configuration identitaire particulière plus que d'un comportement sacrilège. Si tout un chacun est susceptible de s'abandonner, à l'occasion, à de tels " péchés ”, il faut être assurément d'une autre " trempe" ou d'une autre conformation pour pouvoir assumer une telle violation de manière aussi définitive que généralisée. D’une certaine façon, il est possible d'inverser la proposition de Will Roscoe mentionnée en introduction : si les transgenres amérindiens ont été désignés comme des sodomiteshermaphrodites, ce n'est pas parce que les Européens ne comprenaient pas ce qu'ils voyaient, c'est qu'ils le comprenaient peut-être trop bien. Ils n'hésitaient pas entre deux figures répertoriées en Europe pour qualifier une configuration étrange mais ils utilisaient ces deux figures pour spécifier et disqualifier ces êtres redoutablement transgressifs. Rencontrer des " berdaches ", c'était avoir sous les yeux la figure du trickster capable en une facétie et un tournemain de vous bouleverser tout un monde, tout un univers de sens et de pouvoir. Les " berdaches " étaient “ contre-nature " moins parce qu'ils contrevenaient à la bi-partition des genres ou encore à " l'hétérosexualité naturelle " comme peuvent nous le faire penser les textes au premier abord, mais bien plus parce qu'ils opéraient un retournement de prestige et de pouvoir culturellement inacceptable.

\section{BIBLIOGRAPHIE}

ANGELINO, Henry, et Charles SHEDD, 1955, "A note on berdache", American Anthropologist, $57: 121-126$.

BLEYS, Rudi, 1995, The Geography of Perversion: Male-to-Male Sexual Behavior Outside the West and the Ethnographic Imagination, 1750-1918. New York, New York University Press.

BOSSU, Jean-Bernard, 1768, Nouveaux voyages aux Indes occidentales : contenant une relation des différens peuples qui habitent les environs du grand fleuve Saint-Louis appelé vulgairement le Mississipi. Paris, Le Jay. 
BOSWELl, John, 1980, Christianity, Social Tolerance and Homosexuality: Gay People in Western Europe from the Beginning of the Christian Era to the Fourteenth Century. Chicago, University of Chicago Press.

CALlENDER, Charles, et Lee KOCHEMS, 1983, "The North American berdache", Current Anthropology, 24 (4) : 443-470.

CHARLEVOIX, Pierre François-Xavier de, 1744, Journal d'un voyage fait par ordre du roi dans l'Amérique septentrionale. Paris, Ganeau.

CORÉAL, François, 1722, Voyages de François Coréal aux Indes occidentales contenant ce qu'il y a vu de plus remarquable pendant son séjour de 1666 jusqu'en 1697. Amsterdam, Frederic Bernard.

COUROUVE, Claude, 1985, Vocabulaire de l'homosexualité masculine. Paris, Payot.

DELIETTE, Pierre, 1934 [1704], "Memoir of De Gannes concerning the Illinois Country", in Theodore Pease and Raymond Werner, The French Foundations: 1680-1693: Collections of the Illinois State Historical Library. Springfield, Illinois State Historical Library, 302-395 .

DELON, Michel, 1977, "Du goût antiphysique des américains", Annales de Bretagne et des Pays de l'Ouest, LXXXIV (2) : 317-328.

DENIG, Edwin, 1961, Five Indian Tribes of the Upper Missouri. Norman, University of Oklahoma Press.

DE PAUW, Cornelius, 1770, Recherches philosophiques sur les Américains ou mémoires intéressants pour servir à l'histoire de l'espèce humaine. Berlin, Duchesne.

DE TONTI, Henri, 1697, Dernières découvertes dans l'Amérique septentrionale de M. de la Sale. Paris, Jean Guignard.

HAUSER, Raymond, 1990, "The berdache and the Illinois Indian tribe during the last half of the seventeenth century", Ethnohistory, 37 (1) : 45-65.

HENNEPIN, Louis, 1683, Description de la Louisiane nouvellement découverte au sud ouest de la nouvelle France par ordre du roy: Avec la carte du pays, les moeurs et la manière de vivre des sauvages. Paris, Sébastien Huré.

—_, 1697, Nouvelle découverte d'un très grand pays situé dans l'Amérique entre le Nouveau Mexique et la mer glaciale. Utrecht, Guillaume Broedelet.

— 1698, Nouveau voyage d'un pays plus grand que l'Europe. Utrecht, Antoine Schouten.

HÉRAUlT, Laurence, 2007, "Transgenre”, in Michela Marzano, Dictionnaire du corps. Paris, PUF, 940-943.

JACOBS, Sue-Ellen, Thomas WESLEY, et Sabine LANG, 1997, Two-Spirit People : Native American Gender Identity, Sexuality and Spirituality. Urbana et Chicago, University of Illinois Press.

LAFITAU, Joseph François, 1724, Moeurs des sauvages amériquains comparées aux moeurs des premiers temps. Paris, Saugrain et Charles-Estienne Hochereau.

LAHONTAN, Louis Armand de, 1704, Mémoires de l'Amérique septentrionale ou les suites des voyages de Mr. le baron de Lahontan. La Haye, Lhonoré.

LANG, Sabine, 1998, Men as Women, Women as Men : Changing Gender in Native American Cultures. Austin, University of Texas Press.

LAQUEUR, Thomas, 1992, La fabrique du sexe : Essai sur le corps et le genre en Occident. Paris, Gallimard.

LAUDONNIÈRE, René Goulaine de, 1889 [1586], “The voyage of M. René Laudonnière”, in Richard Hakluyt, The Principal Navigations, Voyages, Traffiques and Discoveries of the English Nation. Edinburgh, E. \& G. Goldsmid, 407-440. 
LE MOYNE DE MORGUES, Jacques, 1990, “Voyage en Floride : Illustrations”, in Jean-Paul Duviols et Marc Bouyer, Voyage en Floride de Jean Ribaut, René Laudonnière, Dominique de Gourgues, 1562-1567. La Garenne Colombes, Ed. de l'Espace Européen.

LHOUMEAU, Hélène, 2000, Les expéditions françaises en Floride (1562-1568). Paris, thèse de l'Ecole Nationale des Chartes.

MARQUETTE, Jacques, et Louis JOLIET, 1681, Voyage et découverte de quelques pays et nations de l'Amérique septentrionale. Paris, Estienne Michallet.

OLIVIER, Guilhem, 1990, “Conquérants et missionnaires face au 'péché abominable': essai sur l'homosexualité en Mésoamérique au moment de la conquête espagnole”, Caravelle, $55: 19-51$.

RICHELET, Pierre, 1680, Dictionnaire françois, contenant les mots et les choses, plusieurs nouvelles remarques sur la langue françoise. Genève, Jean Herman Widerhold.

ROSCOE, Will, 1987, "A bibliography of berdache and alternative gender roles among North American Indians", Journal of Homosexuality, 14 (3-4) : 81-171.

—, 1998, Changing Ones : Third and Fourth Genders in Native North America. New York, St. Martin's Griffin.

SALADIN D'ANGLURE, Bernard, 1986, "Du foetus au chamane: la construction d'un troisième sexe", Etudes Inuit/Inuit Studies, 10 (1-2) : 25-114.

ST. COSME, Jean-François Buisson de, 1861 [1700], "Lettre de M.J.F. Buisson de St. Cosme", in De Montigny, De St. Cosme, et Thaumur de la Source, Relation de la mission du Missisipi du séminaire de Québec en 1700. New York, Presse Cramoisy de Jean-Marie Shea, 13-45.

STEINBERG, Sylvie, 2001, La confusion des sexes : le travestissement de la Renaissance à la Révolution. Paris, Fayard.

THWAITES, Reuben Gold, 1900, The Jesuit Relations and Allied Documents : Travels and Explorations of the Jesuit Missionaries in New-France, 1610-1791. Cleveland, Burrows.

TOWLE, Evan, et Lynn MORGAN, 2006, "Romancing the transgender native: rethinking the use of the 'third gender' concept”, in Susan Whittle et Stephen Stryker, The Transgender Studies Reader. New York, Routledge, 666-684.

TREXLER, Richard C., 1995, Sex and Conquest: Gendered Violence, Political Order, and the European Conquest of the Americas. New York, Cornell University Press.

WILliamS, Walter, 1986, The Spirit and the Flesh: Sexual Diversity in American Indian Culture. Boston, Beacon Press.

Gender disorder and transgression: french explorers wrestling with american "berdaches" - Laurence Hérault • Université d'Aix-Marseille, Idemec, France • herault@mmsh. univ-aix.fr

From the texts of the French explorers describing "berdaches", the paper tries to understand the way European cultures have conceived the native American transgender experiences. It shows, in particular, that the European labeling of these experiences (sodomite/hermaphrodite) is less due to a confusion in front of an unknown figure in the European cultures (a third gender), than to the recognition of the transgressive character of "berdaches", which reverse the hierarchy of gender. The American transgenders confronted Europeans with a problem less because they contravened the gender bi-partition or the "natural heterosexuality" but much more because they operated a reversal of prestige and power which was culturally unacceptable.

KEYWORDS: berdache, transgender, two-spirit, gender role, transvestism, homosexuality. 